\title{
Study on Evolution Mechanism of Insurance Market Risk under Information Technology Revolution
}

\author{
Ma Jianxin \\ Central University of Finance and Economics, \\ China Institute for Actuarial Science, China, Box 100081 Beijing
}

\begin{abstract}
Advances in information technologies has changed the way humans are interconnected and brought deep revolution in society, economics and culture. Similarly, the change to the insurance industry is non-negligible. The thesis aims to understand the direction of transformation of the industry and the evolution mechanism of internal systemic risks. Looking at the systemic risk of the insurance market from the perspective of a complex network, it is can be discovered that the occurrence of risk should not only focus on individual risk behaviors or risk factors, but more importantly, it should examine the non-linear relationships among individuals within the network, and in particular the emergent risk evolution mechanism leading to risks. In this research, the author builds a market network simulation model based on the evolution mechanism of the complex network of the insurance market in the analysis of the previous section. The author continues to explore the further evolutionary trend of the insurance market when the sales business method changes based on new technology. The model can be used as a case of the analysis of the evolution mechanism of the insurance market risk.
\end{abstract}

Keywords: insurance market, insurance risk, complex network, evolution theory, simulation

\section{INTRODUCTION}

Information technology has changed the modern society to a great extent. In recent years, the emergence of mobile phones, censors and wearable devices have generated astonishing amount of data. Prediction based on big data has become the focus of the whole society. Traditional businesses such as music, sales, media and financial have been shocked.

Similarly, the change to the insurance industry brought by the technology innovations and change of society is non-negligible. The value of insurance business is to manage risk and the core basis is forecasting. The abundant amount of risk data is the foundation of predicting risks accurately. The technology of big data, clouds and web of things make the acquirement of abundant data more easy and efficient. The monopoly position of risk data and the specialization advantage that Insurance companies have enjoyed are facing great challenges from the innovative companies possessing the most advanced data analysis technologies and predicting techniques and the ability to collect data at low cost and high efficiency.

The thesis aims to understand the direction of transformation of the industry and the evolution mechanism of internal systemic risks, under the challenges of the information technologies. The research try to understand the mechanism of action between the inherent attribute of the industry and the external challenges, so as to predict the future development of the industry and the latent risks in a unified theoretical framework, providing a basis for the insurance regulation and cooperation. 


\section{LIMITATIONS OF CURRENT RISK MANAGEMENT METHODS IN THE INSURANCE} INDUSTRY

Nowadays, the risk management techniques adopted is a combination between "microeconomic regulation" and "macroeconomic regulation", with focus on the measurement and control of risks in the operation of individual entities, such as insurers, insurance intermediaries, etc. The existing systemic risks research in the insurance industry is also focused on the identification and regulation of important institutions that have a significant impact on the insurance industry and the financial industry. This idea of risk identification and risk control still rests on the static "mechanism" and "reductionism" that "the whole is the sum of parts". However, when we look at the real insurance industry value network or the insurance market network, we will find that risks do not only come from the risk behaviors of the individual components that make up the network (such as insurance companies, intermediary companies, service providers, government regulation), among other factors, more importantly, as a complex system. The complex non-linear relationship between individuals makes some triggering factors interact with each other, and the effect is continuously enhanced and mutated by feedback. The state of individual nodes and the role of interaction with other nodes change, and this change is constantly spread through the transmission or infecting mechanism between network nodes. When the local transformation formed by a certain number of individuals breaks through the risk threshold, systemic risks will emerge at the entire network level.

The emergence of risk is a dynamic and uncertain behavior, the currently used risk indicators, static mathematical risk analysis models, and so on are not applicable to understanding the evolutionary generation mechanism of risk in the evolution of insurance network systems. At the same time, in the context of rapid technological change, stakeholders in the insurance industry continue to innovate, relying on complex interactions between each other, making new risks emerge in the insurance industry. The theoretical framework and evolution characteristics of complex networks fit to the process, so it is a good research tool. Using complex network theory to study the evolution of insurance risk can not only explore the evolution mechanism, but also measure certain specific risks. The complex network has a relatively complete mathematical theory and logic framework in the system's generation and evolution mechanism. The inherent mathematical characteristics such as topology structure, network robustness, emergence, phase change and order parameters, ect. has theoretical and practical significance for revealing the evolutionary generation mechanism in the system transition of the insurance market network

\section{RISK EMERGENCE AND METRICS ANALYSIS OF COMPLEX NETWORKS}

Complex network theory is an analytical tool for complex systems and the latest theoretical branch of complexity science. Complex networks are composed of nodes and the edges of connected nodes. The nodes represent the elements or components of the system and the edges represent the interactions and relationships between them. In order to further characterize the network, scientists proposed a number of features and measurement methods for the study of network topology and dynamic mechanism, such as degree distribution, average path length, clustering coefficient, betweens, mixing patterns, ect.

Emergence is the fundamental feature of the system. It arises from the interactions between the microscopic components of the system which include both synergistic and nonlinear functions. Regarding to the definition of emergence concepts, researchers have provided many definitions for reference. Holland(1992) believe that the emergence is a complex phenomenon which produces from a simple locality to the overall level of the system. Xiaoqing Gong(2004) pointed out that the emergence is the process in which the various subjects or subsystems 
interact with each other according to certain rules and eventually form a stable overall structure. Zexian Yan(2005) believes that emergence is a system characteristic which is possessed by high-level things as a whole. And the emergence cannot be predicted.

In terms of emergence metrics, Crutchfield(1994) measured from the perspective of operability, and Bar-Yam(2004) used scale diversity. They all made certain degree of exploration. Some scholars also use information entropy to measure emergence. Lijuan Yang(2010) constructed a cumulative residual entropy model for measuring the tail risk of the financial industry and insurance industry. Dionisio(2005) uses information entropy to measure the uncertainty of financial market. Bo Yuan(2011) proposed a entropy model for measuring the risk which based on the fluctuation of stock returns. Qun Zhang(2008) used a three-dimensional entropy model to rank and evaluate a series of investment programs.

\section{EVOLUTION MECHANISM OF INSURANCE MARKET RISK BASED ON COMPLEX NETWORK THEORY}

In the DT era, the way of companies delivery value to customers has changed. The boundaries between producers, middlemen, service providers, and customers have become blurred. The relationship between them is complicated, such as business connections, knowledge and information exchanges, competition and cooperation, equity investments, supervision, and so on. The relationship is nonlinear, open and decentralized which includes both physical connections and virtual ones. Then entire insurance market presents a complex network topology.

In this paper, according to the topological characteristics of complex network, author proposed three types of macro risk of the insurance market:

\section{Risk of the vulnerability of the insurance market network}

When the insurance market is facing impacts, it shows the robustness against random shocks and the fragility of the impact on key nodes. The vulnerability and robustness of the structure are important characteristics of complex network topologies. The vulnerability of a complex network generally means when some nodes or edges in the network are attacked, the overall performance is greatly reduced, and even the network may be collapsed. Robustness, on the other hand, refers to the ability of the network to maintain its function when nodes or edges randomly fails or are deliberately attacked. Due to its internal complexity, complex networks often exhibit robustness to random shocks and vulnerability to small disturbances that impact key links. When the subject in the system mutates under the influence of environmental randomness, the system has a good self-regulating function, maintaining the normal operation of the system and its own structure. However, when encountering a precise blow against a key node's weaknesses, the system's defects are exposed and there is no ability to quickly adjust.

The most obvious example is the behavior of American International Group in the 2008 global financial crisis. The largest insurance company in the workd faced bankruptcy for a timeand eventually split up and was taken over by the U.S. government. AIG's traditional insurance business is large and healthy. No matter whether it faced the financial crisis in 2008 or a largescale disaster accident in the past, the traditional insurance business has never been seriously affected. This reflects the robustness of its system. The burden which is really dragging the company is the large number of OTC financial derivatives. These innovative business seriously mismatch between its income and risk. Even though the total amount of derivatives transactions is not high in AIG's revenue, the risk losses caused by it are difficult to estimate during the economic period of risk exposure. 
With the development of financial innovation, the hidden systemic risks caused by such small disturbances have not yet been fully identified. For example, the credit insurance business of P2P online loan is difficult to estimate for the hidden risks because of the imperfection of underwriting targets and mechanisms. The risk maybe spread to the insurance companies and whole financial systems through the hooking of financial services. The serious defects in the internal mechanism are the major sources of system vulnerability under changes in the economic environment.

\section{Emergence of risk accidents in insurance market network}

For complex networks, the reliability of an individual node is not consistent with the security of the overall system. "security" and "risk accident" both are phenomena of emergence for a network system. They are not simple superposition of individual attributes of system components. For a simple system, security may be equal to a linear combination of individual component's reliability. Meanwhile, complex system security is a complicated, non-linear coupling and coordination between components and environment which is a overall feature emerged from the whole level. The occurrence of risk accidents in complex systems presents in two basic ways. The first way is that a individual component riggers the risk state, then the risk is transmitted or spread to other components. When a certain amount of individuals arouse a synergistic effect which results in mutations that cannot be self-adjusted and repaired in the system, risk accidents emerge from the whole. The second way is that the component individuals are in certain reliable behavior pattern, but the impact of external environment or the interaction between the components changed. The variation of the relationship promotes the system. The organizations adjust their own behavior, mutate their attributes, change their relationship with other elements and environment. In this way, the degree of variability are constantly enhanced, resulting in changes in the system's topology and functions. In these processes, if the system triggers a risk threshold, "risk accident" will emerge.

For example, the development of big data technology maybe cause the risk of the insured is no longer random. In some cases, when the individual data is easily available and sufficient, the individual risk can be accurately determined, and even the risk mechanism may be discovered. Risks can be promptly intervened and prevented through certain methods. The foundation of insurance business lies on risk aggregation and the law of large numbers. The development of data mining technology may make this foundation disappear, and the business protecting customers from risk loss will be transformed from a financial arrangement such as insurance to a targeted risk management program or service. However, the provider of professional risk management solutions may not be a traditional insurance company, but a highly skilled technical or innovative company in various industries or fields. Traditional insurance business, especially property insurance business, may be weakened or shrunk. When the insurance industry's business paradigm changes dramatically, the original professional company may become a non-professional company because of outdated technology and is gradually marginalized. These scenarios take place on the basis of the premise that the traditional companies are in compliance with the regulations and the risk of the company is controllable in common view. The relationship between the subjects is changed, then some discarded subjects have lost their value in the business network and become dead ends in the system.

In the process of deconstructing and reconstructing the business network, it is very likely that the complex insurance market network has undergone a structural phase change. The system is in a highly unstable state during the phase change period. In this process, the uncertainty such as business risks, compliance risks, market risks, and technical risks will continue to emerge due to the risk concealment of innovative issues. If the migration of the business value network causes the large-scale bankruptcy of existing small insurance companies or the 
operating difficulties of key insurance companies, it maybe will trigger systemic risks in the whole financial industry.

\section{Continuous variability in the risks of the insurance industry during the period of rapid changes in information technology}

The nodes of a complex network are also complex nonlinear systems. When the external environment impacts the system, how the risk mechanism works and conducts is extremely uncertain and difficult to recognize in a short time. As a complex system, the insurance market exhibits the same characteristics when subjected to external shocks. For the innovative issues in the insurance market, the sources, trigger conditions, and conduction paths of risks are all hidden. What often happens is that the hidden risks will be perceived after a risk event occurs. With the rapid changes in current information technology, new technologies emerge in endlessly. The external environment continues to change and technical shocks continue to occur, leading to gradual changes and accumulation of newly unrecognized risks in the insurance market. As the technology and business paradigm are undergoing rapid changes, the speed of new risks changing are also fast. While various technological innovations and theoretical innovations need to be stable for a period of transition, the development and maturation of risk measurement models will also take some time. An excessively rapid change in risk can cause the risk to mutate again before the law of new risks is fully observed. In this process, the risks that have not been eliminated may accumulate, affecting the direction of further evolution of the system.

The evolution of complex networks is path-dependent and irreversible. In the evolution process, the change of any factor or role relationship will lead to generation of different results, with a high degree of uncertainty. When the phase transition of the system occurs and the system moves from one state to another, it cannot return to the original architecture. Therefore, it is very important to make forward-looking judgments on the risk evolution of the insurance market.

\section{EVOLUTION MODELS OF INSURANCE MARKET RISK BASED ON MARKET CONCENTRATION}

The insurance market complex network consists of market players and their couping relationships. For different issues, the construction of the model can take into account different subjects and their specific relationships. The model in this section focuses on the impact of insurance sales transformation which has already occurred. The simulation model attempts to explain how the factors (such as the genetic structure and innovative processes of the insurance company, the construction and distorting mechanisms of agents in market channels, consumer spending habits, and purchasing decisions, etc.) interact and evolve into a new market structure. The model can be used as a special case of risk evolution mechanism analysis.

\section{Analysis of the degree of the concentration of the insurance market network}

Degree distribution is an important indicator of the complex network topology structure, which reflects the degree of network concentration. Under the assumption that each consumer node represents the same amount of premium, the degree of nodes can be expressed as premium or market share of each insurance company. In the current scale-free network research, two most common distributions are exponential distribution $P(k) \sim e^{k}$ and power law distribution $P(k) \sim k$. Figure 1 shows premium income in Chinese insurance market from 2006 to 2015. 


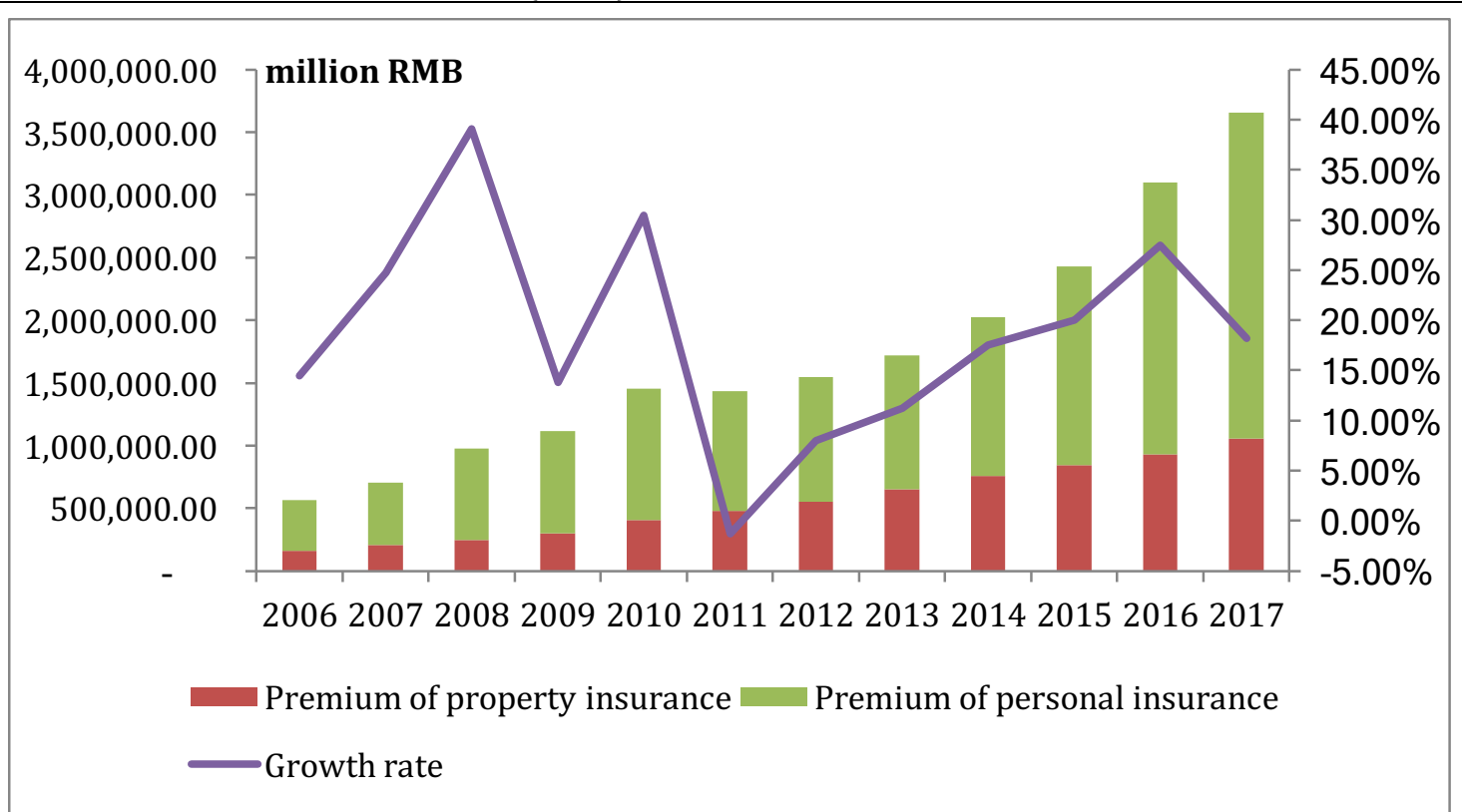

Figure 1: premium income in Chinese insurance market from 2006 to 2015

It can be seen that the premium of Chinese insurance premium has increased year after year. Especially in recent years, premium income has grown rapidly.

The model of this paper is based on the discussion of the Chinese property insurance market. Figure 2 as follows shows the image of Chinese property insurance companies ranked by premium of insurance income in 2015, which uses as the comparison of the model result.

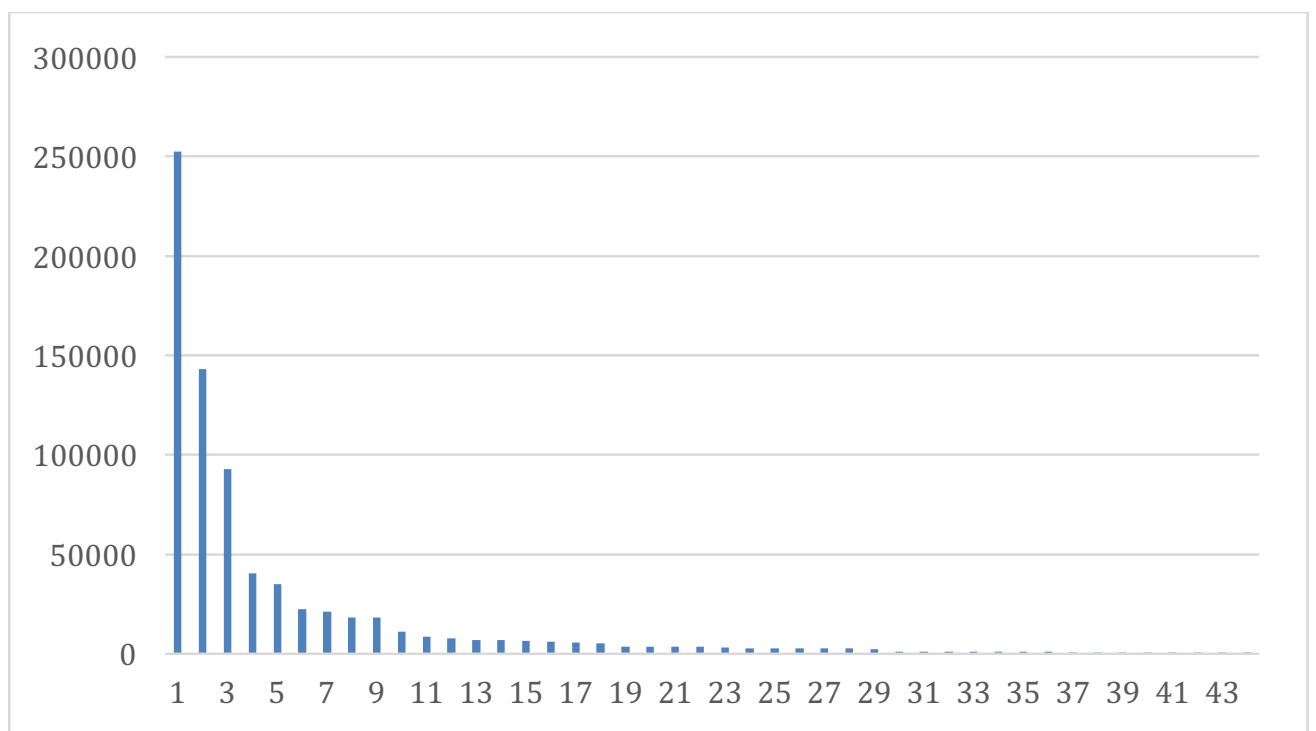

Figure 2: Chinese property insurance companies ranked by premium of insurance income in 2015

Though the result of the fitting calculation of the probability distribution, it can be concluded that the market share of Chinese property insurance companies shows the characteristics of the power law distribution, which means that the insurance market is a scale-free complex network. The power law index of the distribution is 1.70, indicating that the network is highly concentrated. Draw a log-log plot using the cumulative distribution method and perform a linear fit (Figure 3). The calculated variance is 0.963 which means the degree of fit is good. 


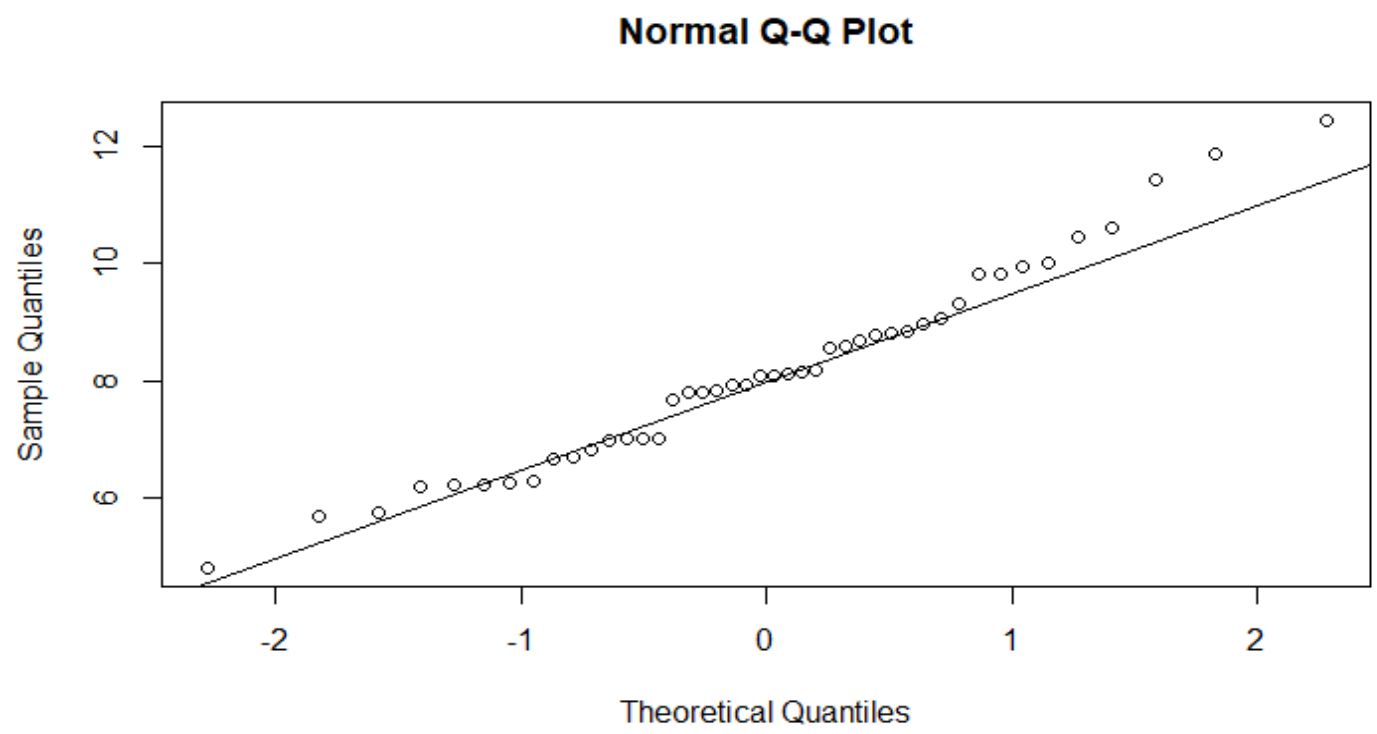

Figure 3: The power law index of the degree distribution of Chinese property insurance network

\section{Construction of the simulation model}

Construct a three-mode network model that includes insurance companies, agency channels, and consumers. Companies include insurance companies or reinsurance companies who have legal operating licenses. Agency channels include professional intermediary agencies, large scale Internet portal sites, e-commerce platforms, personal agents, and concurrent sales agents. Consumers are the one who pay for the insurance. These three types of nodes constitute two levels of the insurance market network. Each consumer chooses at least one purchase channel or insurance company to purchase the insurance service. There is a fierce competition between the market subjects who share resources and clients together.

Network nodes have certain genetic attributes and reflect the characteristics of themselves, such as innovation abilities of the insurance companies, the capabilities of professional services, or consumer preferences. The link between different nodes characterize the purchase relationship of the product or the affiliation of the service. According to the link relationships, the degree of network concentration can be calculated, and its topology and evolution rules can be discussed.

According to the previous analysis, the Chinese property insurance market network has a power law distribution and the simulation can be generated by using the classical BA (Barabasi-Albert) model. In spite of the existed classical mechanisms such as growth and prioritization, two new ideas of "the more suitable the richer" and metabolism are added to the model. "The more suitable the richer" means that the ability of a network node to attract other nodes depends not only on its node degree, but also on the characteristics of the node itself, such as innovation ability, competitiveness, possession of resources, or the professional quality, ect. Using the genetic properties and the degree of the nodes to construct the fitness function that attract other nodes, the higher the fitness function is, the stronger the ability of attraction. "Metabolism" refers to the life cycle of a node, not only considering the node's "birth" but also its "death".

\section{Insurance market network based on the simulation model}

The simulation program runs a total of 36 time units. The market share of the insurance companies is be calculated and recorded once a time unit. Figure 4 shows the simulation 
insurance market network which includes the nodes of insurance company, agency company, agent individual, and consumers at the $25^{\text {th }}$ cycle which according to 2015 in reality.

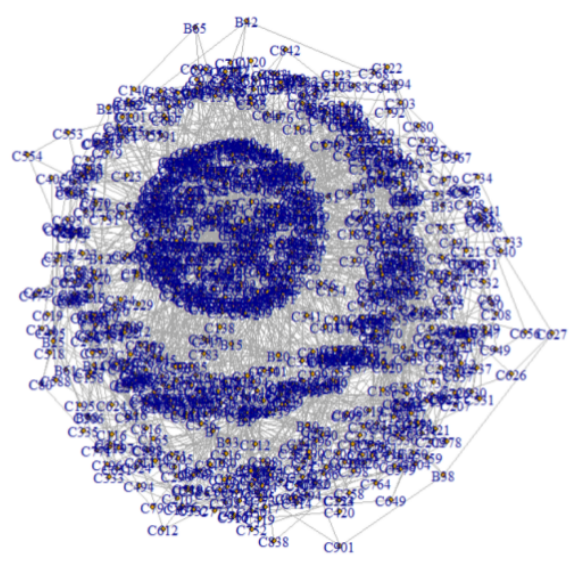

Figure 4: The simulation insurance market network in 2015

\section{Comparison between Evolutionary Model and Real Insurance Market}

Figure 5 shows the simulation companies ranked by simulation market share of the insurance market complex network at the $25^{\text {th }}$ cycle unit which according to 2015 .

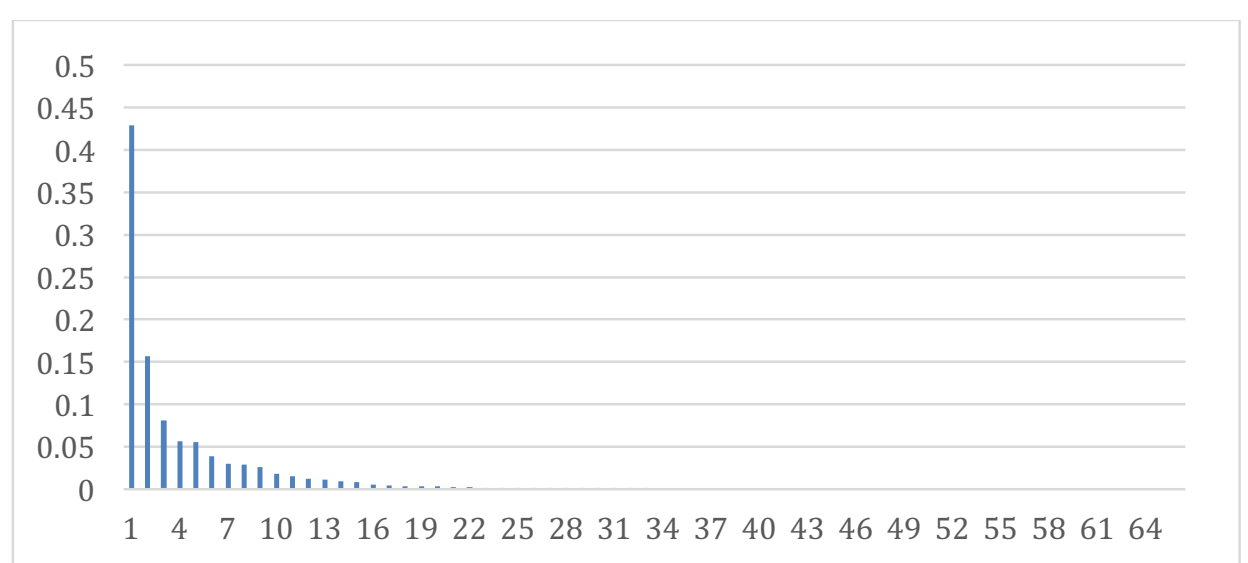

Figure 5: Simulation property insurance companies ranked by market share in 2015

Using R software, the exponent of the power law distribution can be calculated which shows $=1.76$. Draw a log-log plot using the cumulative distribution method and perform a linear fit (Figure 6). The calculated variance is 0.847 which means the degree of fit is good. It can be considered that the stimulation insurance market in 2015 compliance a power law distribution whose law index is 1.76 . The market concentration of the simulated market complex network is close to the real situation, which shows that the market network model we have constructed has certain explanatory capacity to the reality. 


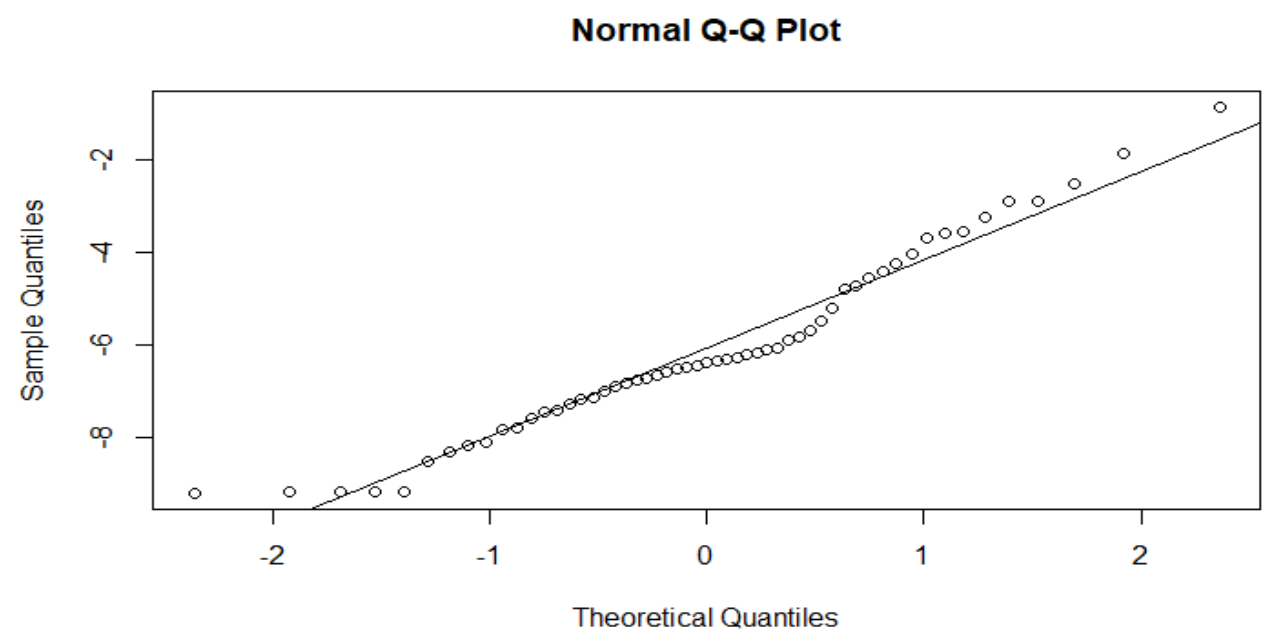

Figure 6: The power law index of the degree distribution of Chinese property insurance network

The Evolution of the Structure of Insurance Market Network under Transformation of Sales Method

\section{Analysis of the impact of transformation of sales method based on new technology}

The Simulation model established above can be used to study the impact of a new insurance sales method. In the benefit of the development of mobile technology, some integrated mobile applications with a high usage rate have also started to open exclusive channels for insurance sales. In China, two important daily applications, Alipay and WeChat, are providing new ways to purchase insurance products.

The user can conveniently open a common application on the mobile phone and click on the button marked "Insurance Services". A lot of insurance products from dozens of companies appear at the same time. People can search for matching products according to their own needs. The similar products of each company is placed on the same page for customers to choose. Users can easily and directly compare various insurance products which provide similar terms with their prices, services, the amount of purchase and the amount of pageviews, etc. Users can also enter related forums to view discussions and analysis of a product which he is interested in. In this process, users can get artificial or intelligent customer service anytime to answer various questions about product. This greatly improves the speed and accuracy of user decision-making, and reduces the information asymmetry brought about by the professionalism and complexity of finance. Most online insurance products support online reporting and online claims for certain or all cases, which greatly save customers' time and makes mobile insurance products more valuable.

What we are interested in is that, when insurance can be purchased on important mobile applications, this change in sales model will promote market competition or otherwise promote the market concentration. In one hand, the change in sales maybe make more small and medium-sized insurance companies which are public unknown before recognized by consumers and reduce the gap in capability of technology and construction of channel teams between the big companies and small companies. On the other hand, the change in sales maybe concentrate more costomers in large insurance companies by "Herd Effect".

In order to find out the effect, we join 2 website sales nodes with high usage rate at the $28^{\text {th }}$ cycle which according to 2018 in reality, and observe changes in the market structure under the premise whether cuonsumers will change their sales habits or not. 


\section{Only $5 \%$ of consumers are willing to consider new sales methods}

Under the assumption of impact, we can calculate the index of the power law distribution of the insurance market network year by year. The index reflects the concentration of the market. The year-by-year changes of the power law index are shown in Figure 7 below.

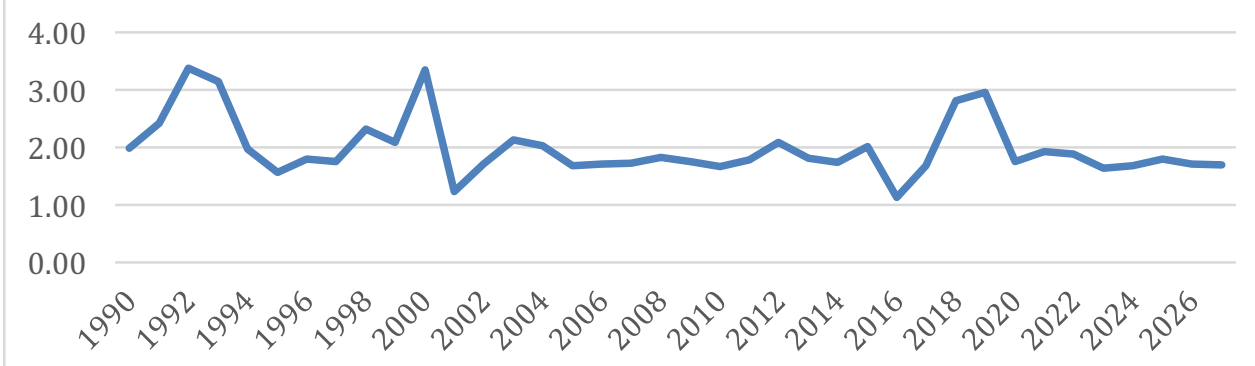

Figure 7: The year-by-year changes of the power law index of the simulation market network distribution based on new sales change and $5 \%$ of customers prefer

Larger power law index of the distribution indicates that the head part of the curve falls more slowly, which means the market concentration that the distribution represents is lower. As can be seen from the figure, after the new sales channel is established, it will have s strong impact on the market structure in a short period of time, then the market concentration will fall back to the level before the impact.

\section{$30 \%$ of consumers are willing to consider new sales methods}

We assume that due to the continuous construction and promotion of new sales channels and the generational changes in consumer groups, there are $30 \%$ of consumers shift from the traditional prudent insurance purchase mode to the active mode of autonomous search and purchase online. Figure 8 below shows the evolutionary trend curve after the manifest superposition of consumer spending habits.

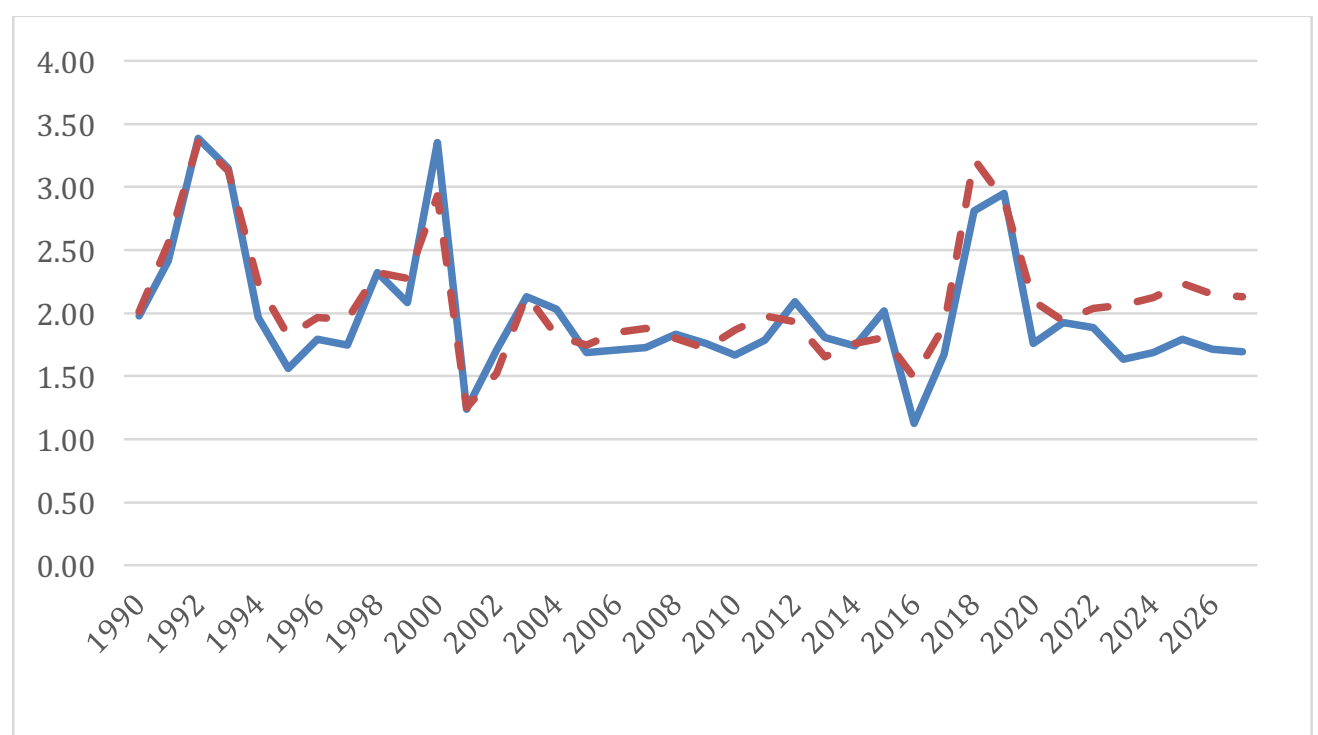

Figure 8: The Comparison of Impact of new sales method to Insurance Market Based on different consumer habits

Intuitively, under the effect of both impacts that favor the circulation of internet insurance products, the concentration trend of the stabilized insurance market structure is lower than that of a single shock. The Wilcoxon rank test is used to test whether the evolution of the market structure has actually changed since there is a obviously changes in consumer spending habits. 
Assume the sequence of the degree of concentration of the simulated insurance market when $5 \%$ of consumers purchase habits change is $M_{1}$, while the sequence of the concentration in the other case which means $30 \%$ of consumers purchase habits change is $M_{2}$ 。

Hypothesis testing questions : $H_{0}: M_{1}=M_{2} \quad H_{1}: M_{1}<M_{2}$

Using R software to calculate, the test p-value is 0.001953 . Under the $99 \%$ confidence level, the original hypothesis is rejected and the alternative hypothesis is accepted. After the consumer's consumption habits are significantly changed, the market concentration is obviously reduced. The decline in market concentration indicates that the market monopoly in the past has been further broken down and the market has become more competitive. This shows that in the case of Ailpay or WeChat, the phenomenon-level mobile applications cutting into the front end of insurance sales, it can significantly changed the value distribution principle in the insurance market, and has enabled many small-sized companies who has no capability to build a traditional agent team to obtain more market opportunities.

\section{CONCLUSION}

This paper analyzes the systematicity and complexity of the insurance market, demonstrates that the insurance market is a typical complex system. On this basis, the topological characteristics of complex networks are used to study the change trend of the insurance market under the impact of information technology innovation. The evolution mechanism of the macro risk of the three types of insurance market network system is discussed. From the perspective of the essential structure and evolution law of the insurance market, the emergence and classification of the macro-risk of the insurance market is forecasted, which provides an effective theoretical tool for the study of insurance market risk in the rapidly changing period full of uncertainty.

\section{Reference}

Jinglong Guo, Qian Zhao.(2014), A Literature Review of Systematic Risks in Insurance, Insurance Research, 2014(6):42-52

Lijuan Yang, Xingsi Li(2010), Residual entropy model for measuring tail risk[J], Operations and Management , 2010,19(6):98-103

Bo Yuan, Jianguo Wang(2011), Research on Stock Entropy Risk Measurement Method[J], Chinese Journal of Engineering Mathematcs, 2011,28(3):401-405

Qun Zhang, Wensheng Liu(2008), Risk-based decision making based on three dimensional entropy measurement model, Statistics and Decision, 2008(16):60-62

Qiaoqing Gong(2004), The emergence and evolution of economic systems-from the complexity science point of view[J],Financial Review, 2004,5(9):12-18

Zexian Yan(2005), A new way of highlight problem research-from the dynamic mechanism[J],Philosophical research, 2005, (7):101-107

Foss N(1997), Resources, firms and strategies, a reader in the resource-based perspective[M], Oxford: Oxford University Press

Geneva Association(2011), Considerations for Identifying Systemically Important Insititutions in Insurance, April 2011

Geneva Association(2013), Group-wide risk and capital management of internationally active insurance groups, April 2013

Geneva Association(2012), Insurance and Resolution in light of the Systemic Risk Debate, February 2012 
Geneva Association(2010), Key Financia Stability Issues in Insurance, July 2010b

Geneva Association(2012), Surrenders in the Life Insurance Industry and their Impact on Liquidity, August 2012

Geneva Association(2010), Systemic Risk in Insurance-An analysis of insurance and financial stability, March 2010a

Geneva Association(2012), The institutional framework for global insurance regulation and supervision: the challenging landscape, Dec 2012

Geneva Association(2013), Variable annuities: and analysis of financial stability, Mar. 2013

Holland J H.(1992) Complex adaptive systems. Daedalus. 1992. 17-30

Crutchfield J.(1994) The calculi of emergence: Computation, dynamics, and induction[J], Physica D,1994,75(13):11-54

Bar-Yam Y.(2004) A mathematical theory of strong emergence using multiscale variety[J], Complexity,2004,9(6):15-24

Dionisio A., Menezes R. M.(2005), Endes D A. Uncertainty analysis in financial markets: can entropy be a solution[M], University of Essex, UK, 2005, 6

Jackson M, Watts A(2002), The evolution of Social and Economic Netwoks[J], Journal of Economic Theory 106:265_295

Harrington(2009), The financial crisis, systemic risk and the future of insurance regulation, Journal of Risk and Insurance, 2009 